Check for updates

Cite this: Chem. Sci., 2019, 10, 8981

๑ All publication charges for this article have been paid for by the Royal Society of Chemistry

Received 11th June 2019

Accepted 5th August 2019

DOI: $10.1039 / c 9 s c 02851 j$

rsc.li/chemical-science

\section{Understanding the structure and dynamics of hydrogenases by ultrafast and two-dimensional infrared spectroscopy $\dagger$}

\author{
Marius Horch, (D) *ab Janna Schoknecht, ${ }^{\mathrm{b}}$ Solomon L. D. Wrathall, ${ }^{a}$ \\ Gregory M. Greetham, ${ }^{c}$ Oliver Lenz $\mathbb{D}^{b}$ and Neil T. Hunt (D) ${ }^{a}$
}

\begin{abstract}
Hydrogenases are valuable model enzymes for sustainable energy conversion approaches using $\mathrm{H}_{2}$, but rational utilization of these base-metal biocatalysts requires a detailed understanding of the structure and dynamics of their complex active sites. The intrinsic $\mathrm{CO}$ and $\mathrm{CN}^{-}$ligands of these metalloenzymes represent ideal chromophores for infrared (IR) spectroscopy, but structural and dynamic insight from conventional IR absorption experiments is limited. Here, we apply ultrafast and two-dimensional (2D) IR spectroscopic techniques, for the first time, to study hydrogenases in detail. Using an $\mathrm{O}_{2}$-tolerant [NiFe] hydrogenase as a model system, we demonstrate that IR pump-probe spectroscopy can explore catalytically relevant ligand bonding by accessing high-lying vibrational states. This ultrafast technique also shows that the protein matrix is influential in vibrational relaxation, which may be relevant for energy dissipation from the active site during fast reaction steps. Further insights into the relevance of the active site environment are provided by 2D-IR spectroscopy, which reveals equilibrium dynamics and structural constraints imposed on the $\mathrm{H}_{2}$-accepting intermediate of [NiFe] hydrogenases. Both techniques offer new strategies for uniquely identifying redox-structural states in complex catalytic mixtures via vibrational quantum beats and 2D-IR off-diagonal peaks. Together, these findings considerably expand the scope of IR spectroscopy in hydrogenase research, and new perspectives for the characterization of these enzymes and other (bio-)organometallic targets are presented.
\end{abstract}

\section{Introduction}

Dihydrogen $\left(\mathrm{H}_{2}\right)$ is a clean fuel that releases large amounts of free energy but no greenhouse gases upon combustion. Catalysing the reversible cleavage of $\mathrm{H}_{2}$, hydrogenases are valuable model enzymes for sustainable energy conversion approaches. ${ }^{1}$ Their utilization as biotechnological targets or blueprints for bio-inspired chemistry, however, requires a thorough understanding of the structural, dynamic, and mechanistic traits of these metalloenzymes. Several central aspects of hydrogenase function are still far from understood, and, thus, there is a demand for new spectroscopic techniques that can reveal ever greater levels of molecular detail.

Infrared (IR) spectroscopy has played a central role in the characterization of hydrogenases since the first detection of

${ }^{a}$ Department of Chemistry, York Biomedical Research Institute, University of York, Heslington, York, YO10 5DD, UK. E-mail: marius.horch@york.ac.uk

${ }^{b}$ Institut für Chemie, Technische Universität Berlin, Straße des 17. Juni 135, Berlin, D10623, Germany

'STFC Central Laser Facility, Research Complex at Harwell, Rutherford Appleton Laboratory, Harwell Science and Innovation Campus, Didcot, Oxford, OX11OPE, UK $\dagger$ Electronic supplementary information (ESI) available: Supplementary figures. Description of the Morse fit. Analysis of $\mathrm{CN}$ stretch vibrational relaxation. References. See DOI: 10.1039/c9sc02851j biologically unprecedented $\mathrm{CO}$ and $\mathrm{CN}^{-}$ligands in the active sites of these enzymes (Fig. 1, top). ${ }^{2-5}$ Both types of ligands exhibit structurally sensitive bond stretching vibrations in an otherwise featureless region of the IR spectrum (Fig. 1, top). ${ }^{3,6}$ Thus, IR spectroscopy has been widely used to monitor transitions between redox-structural states of hydrogenase active sites under various experimental conditions ${ }^{7,8}$ So far, however, all IR studies on these enzymes have focused on linear absorption experiments and the interpretation of vibrational frequencies. Due to the strict localization of $\mathrm{CO} / \mathrm{CN}$ stretch modes and the multitude of determinants that govern their vibrational frequencies, structural information from this single set of observables is inherently limited. Moreover, time-resolved insights into hydrogenases are still scarce. While nonequilibrium studies have provided information on active site state interconversions, ${ }^{9}$ they fail to provide dynamic insights into the states themselves, as would be obtained under equilibrium or steady state conditions. Thus, individual redoxstructural states have only been characterised in a timeaveraged manner so far.

Here, we apply ultrafast pump-probe and two-dimensional (2D) IR techniques, for the first time, to study the active site of a hydrogenase. Using ultrashort laser pulses and multiple lightmatter interactions, these non-linear experiments provide access 


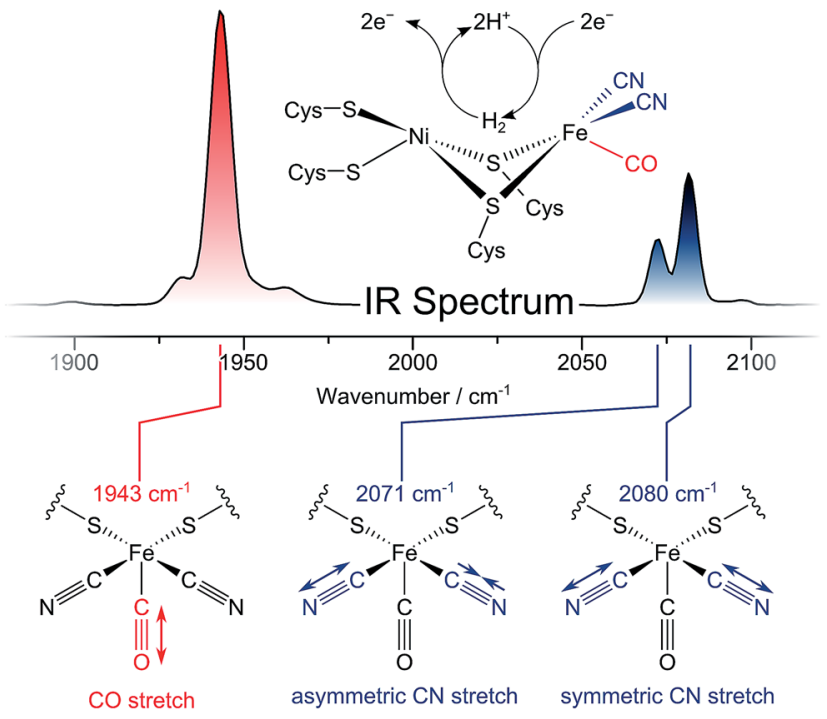

Fig. 1 (Top) IR absorption spectrum and active site structure of oxidized $\mathrm{ReRH}$ in the $\mathrm{Ni}_{\mathrm{a}}$-S state, the $\mathrm{H}_{2}$-accepting intermediate of [NiFe] hydrogenases. (Bottom) Visualization of $\mathrm{CO}$ (red) and $\mathrm{CN}$ (blue) stretching modes detected in the IR spectra of [NiFe] hydrogenases. Vibrational frequencies of these modes are sensitive to details of the active site, so that each redox-structural [NiFe] state exhibits a distinct signature of three absorption signals. Listed Frequencies refer to the $\mathrm{Ni}_{\mathrm{a}}-\mathrm{S}$ state of $\mathrm{ReRH}$.

to several unexplored observables that enhance our understanding of the structure and dynamics of hydrogenases under biologically relevant conditions..$^{10-12}$ To illustrate the merit of ultrafast IR techniques, we utilize the regulatory [NiFe] hydrogenase from Ralstonia eutropha (ReRH), which proved to be a valuable model system. ${ }^{13-15}$ Like other [NiFe] hydrogenases, ${ }^{7}$ this enzyme harbours a heterobimetallic active site containing two metal ions, $\mathrm{Ni}$ and $\mathrm{Fe}$, that are coordinated by four cysteine thiolates (Fig. 1, top). ${ }^{13,14,16-18}$ In addition, the low-spin $\mathrm{Fe}^{\mathrm{II}}$ ion (S $=0)^{14}$ carries one $\mathrm{CO}$ and two $\mathrm{CN}^{-}$ligands, as observed for all known [NiFe] hydrogenases. ${ }^{7,19-23}$ Being an $\mathrm{H}_{2}$-sensing hydrogenase, $R e$ RH exhibits comparably low catalytic activity for both $\mathrm{H}_{2}$ activation and evolution, ${ }^{17}$ most likely due to unusual properties of its FeS clusters. ${ }^{14}$ On the other hand, ReRH is not inhibited by dioxygen $\left(\mathrm{O}_{2}\right),{ }^{16,17,24}$ and only few (virtually pure) catalytically active species are observed under most relevant conditions. ${ }^{13,16-18}$ Specifically, the as-isolated, oxidized form of the enzyme resides in the active $\mathrm{Ni}_{\mathrm{a}}-\mathrm{S}$ state $\left(\mathrm{Ni}^{\mathrm{II}}, \mathrm{S}=0\right),{ }^{13,16-18}$ which is characterized by an open binding site between nickel and iron..$^{13,14,25}$ In the current study, novel insights into this $\mathrm{H}_{2}$-accepting catalytic intermediate of [NiFe] hydrogenases are provided. Using ultrafast and 2D-IR spectroscopies, we shed light on key bond properties of the $\mathrm{CO}$ ligand, dynamic interactions between the diatomic ligands, and the complex interplay between the [NiFe] active site and its protein environment.

\section{Results and discussion}

The $R e \mathrm{RH}$ active site produces an IR absorption spectrum containing three peaks (Fig. 1, top) at 1943, 2071, and $2080 \mathrm{~cm}^{-1}$ that have been assigned to the fundamental transitions (ground to first excited state; $v=\mathbf{0 - 1}$ ) of one $\mathrm{CO}$ and two CN stretching vibrations (Fig. 1, bottom; Fig. 2).,5, ${ }^{4,136-18}$ IR pump-probe spectra are generated by successive interaction of the sample with two broadband $\left(>300 \mathrm{~cm}^{-1}\right)$ femtosecondduration IR laser pulses. The pump pulse excites all molecular vibrations within the bandwidth of the laser, and the response of the sample to this perturbation is monitored by the probe pulse as a function of pump-probe delay time. IR pump-probe spectra are presented as difference spectra (Fig. 3, top) reflecting the change of probe light absorption by the sample following interaction with the pump pulse.

In the region of CO stretching vibrations $\left(1850-2000 \mathrm{~cm}^{-1}\right)$, the IR pump-probe spectrum of oxidized $R e \mathrm{RH}$ contains multiple peaks (Fig. 3A). A negative feature at $1943 \mathrm{~cm}^{-1}$ is assigned to bleaching and stimulated emission of the $v=\mathbf{0 - 1}$ transition of the CO stretching vibrational mode (Fig. 1 and 2). This signal arises from population of the first excited state $(v=$ 1) by pump pulse excitation. The same process gives rise to enhanced absorption (positive peak) at $1918 \mathrm{~cm}^{-1}$ which is assigned to the $v=1-2$ transition of the CO stretching vibration. Likewise, smaller positive features at 1894 and $1870 \mathrm{~cm}^{-1}$ are assigned to the $v=2-3$ and $v=3-4$ transitions, following population of the second and third vibrationally excited state, respectively (Fig. 2 and 3A). ${ }^{26,27}$

Through observation of transitions from higher lying vibrational states $(v>1)$, IR pump-probe spectroscopy allows

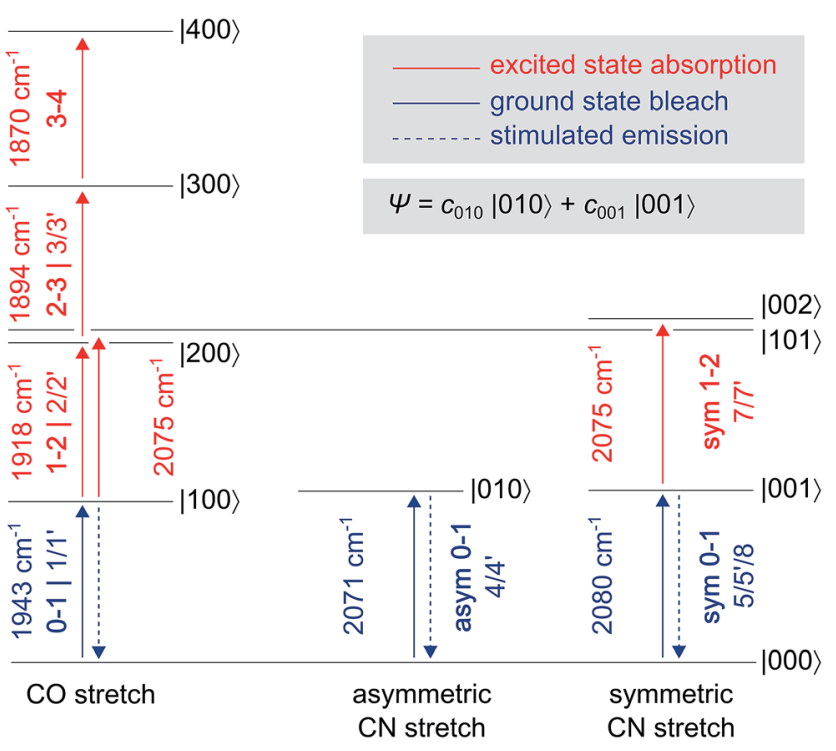

Fig. 2 Eigenstates $|a b c\rangle$ and transition energies (drawn to scale) of the $\mathrm{CO} / \mathrm{CN}$ stretch vibrational manifold, as detected for oxidized ReRH in the $\mathrm{Ni}_{\mathrm{a}}-\mathrm{S}$ state. $a, b$, and $c$ refer to the number of vibrational quanta in the $\mathrm{CO}$ stretch mode, the asymmetric $\mathrm{CN}$ stretch mode, and the symmetric $\mathrm{CN}$ stretch mode, respectively. As discussed in the main text, a common ground state $|000\rangle$ can be assumed. Transitions are labelled with the signal descriptors used in the pump-probe (Fig. 3) and 2D-IR (Fig. 6) spectra. Note that 2D-IR signal 6 is not included as it has contributions from several transitions, and the associated eigenstate energies are not precisely known (Fig. S4 $\uparrow$ ). A non-stationary (coherent) state representing a linear combination of $\mathrm{CN}$ stretch excited states is listed separately. 

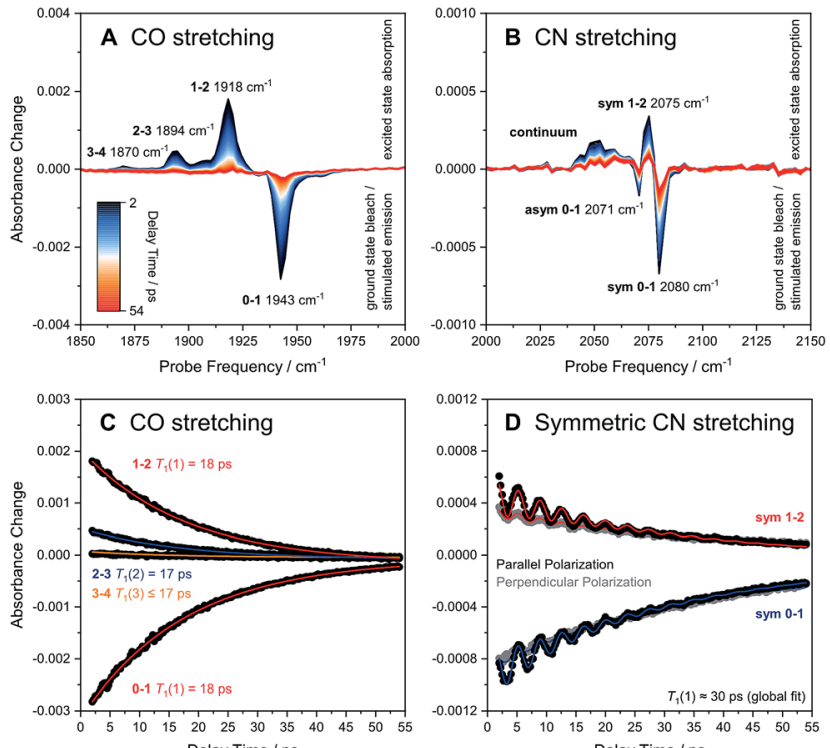

Delay Time / ps

Fig. 3 Broadband pump-probe spectra (A and B) and time evolution of selected signals ( $C$ and D) as obtained for oxidized $R e R H$ in the $\mathrm{Ni}_{a}-S$ state. Data referring to $\mathrm{CO}$ and $\mathrm{CN}$ stretch modes are plotted separately on the left ( $A$ and $C$ ) and right ( $B$ and $D)$, respectively. Unless indicated otherwise, all data were acquired with a parallel pumpprobe polarization geometry. $\$$ Transitions between vibrational eigenstates $|m\rangle$ and $|n\rangle$ are labelled as $\mathbf{m}-\mathbf{n}$. For the $\mathrm{CO}$ stretch mode (C), vibrational lifetimes $T_{1}(n)$ of eigenstates $|n\rangle$ were obtained by fitting monoexponential decay curves (coloured lines) to experimental $\mathbf{m}-\mathbf{n}$ time traces (black dots). For the symmetric $\mathrm{CN}$ stretch mode (D), $T_{1}(1)$ was determined by globally fitting the sum of a monoexponential decay curve and a monoexponentially damped sine function (coloured lines) to experimental 0-1 and 1-2 time traces obtained with both parallel (black dots) and perpendicular polarization (grey dots). sym, symmetric $\mathrm{CN}$ stretch mode; asym, asymmetric $\mathrm{CN}$ stretch mode.

experimental insights into the potential energy surface along the $\mathrm{CO}$ bond coordinate that go beyond IR absorption methods. Due to the constant spacing between transition energies (i.e. signals in the pump-probe spectrum), the metal-bound and protein-embedded CO ligand can be treated as an anharmonic diatomic molecule (see SI $2 \dagger$ ). Fitting a Morse potential ${ }^{28}$ to the experimentally observed transition energies, we obtain direct insights into fundamental properties of the $\mathrm{CO}$ ligand including bond strength and anharmonicity (Fig. 4). As a Lewisamphoteric ligand, ${ }^{29,30} \mathrm{CO}$ is capable of balancing the electron density at the active site, which is likely relevant for $\mathrm{H}_{2}$ binding and activation as well as the tuning of ground and transition state energies of species containing bridging hydrides. Thus, these insights into $\mathrm{CO}$ bonding promise to further our structural understanding of the [NiFe] active site and its rearrangement during catalytic turnover. In addition, these observables can be used as new structural markers to complement the conventional analysis of fundamental vibrational frequencies in hydrogenase research. Finally, the Morse fit also reveals the harmonic vibrational frequency, i.e. the quantity that is typically calculated in, e.g., density functional theory (DFT) studies. Remarkably, this frequency is higher than the experimental transition energy by $24 \mathrm{~cm}^{-1}$. This significant effect has not

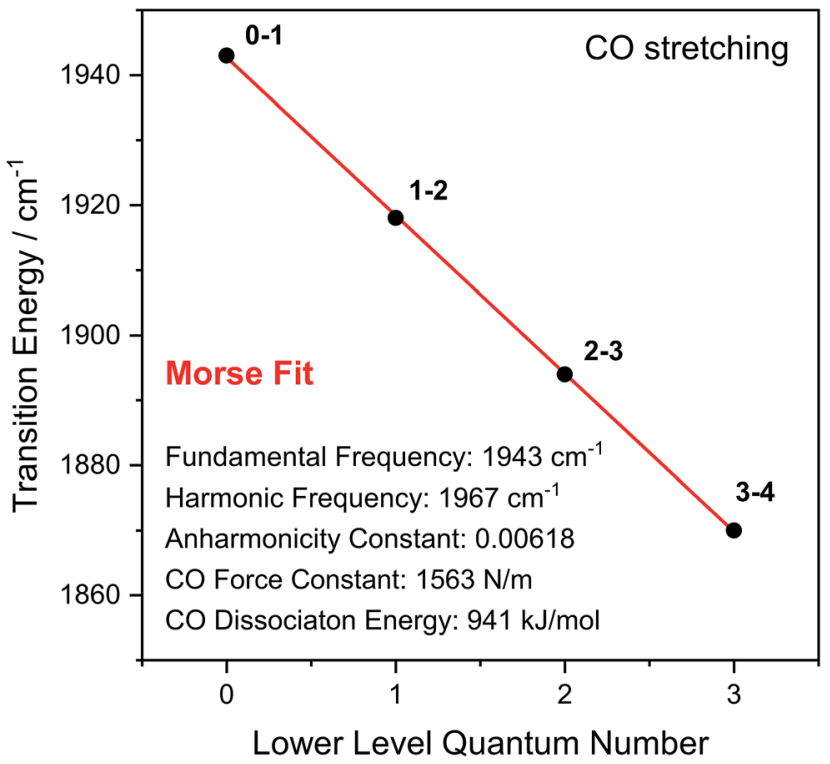

Fig. 4 Transition energies observed for the $\mathrm{CO}$ stretch vibration of oxidized $\mathrm{ReRH}$ in the $\mathrm{Ni}_{2}-\mathrm{S}$ state. Indicated bond properties were determined by fitting a function representing energy level separations of a Morse potential (red line) to the experimental data (black dots). Transitions between vibrational eigenstates $|m\rangle$ and $|n\rangle$ are labelled as $\mathrm{m}-\mathrm{n}$. For details, see $\mathrm{SI} 2 \dagger$

been considered in any of the previously published theoretical studies on hydrogenases, which has far-reaching implications for the future implementation and interpretation of theoretical vibrational analyses in hydrogenase research.

IR pump-probe spectra obtained at higher frequencies $\left(2000-2150 \mathrm{~cm}^{-1}\right)$ reveal negative peaks that can be assigned to ground state bleaching and stimulated emission (0-1) of the asymmetric $\left(2071 \mathrm{~cm}^{-1}\right)$ and symmetric $\left(2080 \mathrm{~cm}^{-1}\right) \mathrm{CN}$ stretching vibrational modes (Fig. 1 and 3B). While transient absorption from the first excited state (1-2) is clearly resolved for the symmetric stretching mode at $2075 \mathrm{~cm}^{-1}$ (Fig. 2 and 3B), the corresponding signal of the asymmetric mode is obscured by a broad continuous feature between $c a .2040$ and $2070 \mathrm{~cm}^{-1}$. Due to strong overlap of the closely spaced and apparently less anharmonic $\mathrm{CN}$ stretch vibrations, these transitions will be discussed in more detail on the basis of 2D-IR spectra (vide infra).

The time-evolution of signals in the pump-probe spectra provide information on the lifetimes of vibrationally excited states $\left(T_{1}\right)$. These quantities reflect the rate of vibrational energy transfer away from the active site, thereby providing novel vibrational markers that yield insights into the dynamic active site environment. Apart from one known exception, ${ }^{31} \mathrm{CO}$ and $\mathrm{CN}$ stretch frequencies are only sensitive towards isotope exchanges on the diatomic ligands themselves, ${ }^{4,5}$ due to the highly localized nature of these normal modes. In contrast, $T_{1}$ may be highly sensitive towards substrate, protein, or solvent isotope exchanges, ${ }^{32-35}$ thereby allowing direct structural insight beyond the diatomic ligands. The intensities of both the $\mathbf{0 - 1}$ and 1-2 signals in the CO probe region of the spectra (Fig. 3A) 
are well modelled by mono-exponential decay curves (Fig. 3C), revealing an upper limit of $18 \mathrm{ps}$ for the $v=1$ state lifetime (Fig. 3C). This small value reflects fast intramolecular energy dissipation upon $\mathrm{CO}$ bond distortion, which could be relevant in case of fast catalytic steps that would be otherwise hampered by barrier recrossing. For a harmonic oscillator that is linearly coupled to a harmonic bath, vibrational lifetimes decrease linearly with the vibrational quantum number, ${ }^{36}$ as observed for a mononuclear homoleptic metal carbonyl compound in solution. ${ }^{26}$ In contrast, higher excited states of the CO stretch mode of $R e \mathrm{RH}$ decay at comparable rates, indicating considerable deviation from this relaxation model.

Notably, the CO vibrational lifetime of ReRH differs from most previously studied synthetic carbonyl and cyanido complexes. ${ }^{26,27,33-35,37-41}$ The observed value of 18 ps is significantly shorter than those typically observed for synthetic systems in non-interacting or hydrophobic solvents ${ }^{39,41}$ but longer than those of metallocarbonyls studied in water. ${ }^{35}$ Since energy dissipation from the deeply buried active site cannot proceed via collision with solvent molecules, vibrational relaxation likely involves anharmonic coupling ${ }^{42}$ of the CO stretch coordinate through $\sigma$ or $\pi$ bonding interactions with the Fe ion, as observed for comparably fast-relaxing carbonyl derivatives of heme proteins and related metalloporphyrins. ${ }^{43}$ In line with this proposal, DFT calculations on an [FeFe] hydrogenase model compound revealed pronounced anharmonic coupling between $\mathrm{CO} / \mathrm{CN}$ stretch modes and low-frequency $\mathrm{Fe}(\mathrm{CO})(\mathrm{CN})_{2}$ metalligand vibrations that are observed experimentally for $[\mathrm{Fe}]$, [FeFe], and [NiFe] hydrogenases. ${ }^{13,40,44,45}$ It is thus tempting to propose that the short CO lifetime is due to through-bond energy redistribution towards the protein matrix, possibly involving the hydrogen-bonded $\mathrm{CN}^{-}$ligands (vide infra) or the bridging cysteines. This conclusion highlights the role of the protein matrix in defining active site characteristics and explains the considerably longer relaxation times observed for non-proteic dithiolato-bridged carbonyl compounds in weakly interacting solvents. ${ }^{39,41}$

Due to smaller intensities, strongly overlapping signals of opposite sign, and kinetic complexity (vide infra), the extraction of vibrational lifetimes is more challenging for the two $\mathrm{CN}$ stretch modes (Fig. 3D). First-excited-state lifetimes of both vibrations are on the same order of magnitude but seem to be slightly longer than that of the $\mathrm{CO}$ stretch mode. Based on extensive analyses (see ESI: SI $3 \dagger$ ), we conclude that the apparent lifetime of the symmetric CN stretch mode is $c a .30$ ps (Fig. 3D), and a similar value can be assumed for the asymmetric counterpart. The decay curves of all signals associated with the two CN stretch modes exhibit a pronounced oscillatory modulation, which is best observed after subtracting a mono-exponential background (Fig. 5A). This quantum beat pattern results from coherent excitation of the two CN stretch modes, which creates a superposition of their first excited states. ${ }^{46-48}$ The coherent superposition state decays faster than the individual excited states, indicating that the observed time constant of $10 \mathrm{ps}$ can be associated with its pure dephasing time $T_{2}^{*}$. As expected, ${ }^{46-48}$ the quantum beat frequency $\left(9 \mathrm{~cm}^{-1}\right)$ equals the difference between fundamental transition energies of the two involved
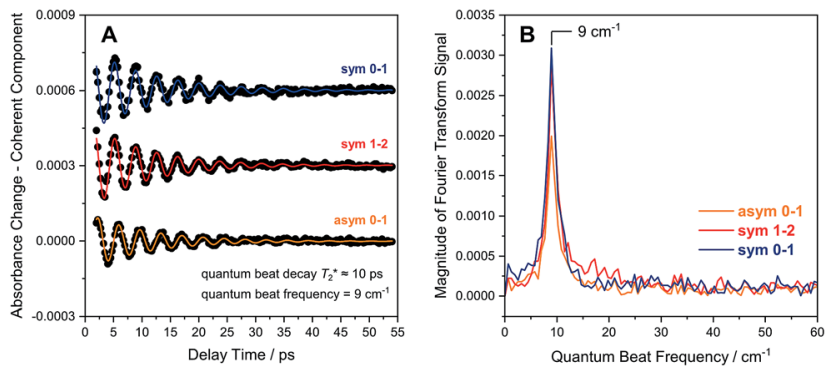

Fig. 5 (A) Quantum beat patterns observed for $\mathrm{CN}$ stretch transitions of oxidized $\mathrm{ReRH}$ in the $\mathrm{Ni}_{\mathrm{a}}-\mathrm{S}$ state. Data are shown stacked, and a monoexponential decay background has been subtracted from the raw data (Fig. 3D) to eliminate non-coherent contributions. Quantum beat frequencies and decay times were determined by fitting monoexponentially damped sine functions (coloured lines) to the experimental data (black dots). (B) Fourier transform of the time traces shown in (A). Data relating to transitions between vibrational eigenstates $|m\rangle$ and $|n\rangle$ are labelled as $\mathrm{m}-\mathrm{n}$. Sym, symmetric $\mathrm{CN}$ stretch mode; asym, asymmetric $\mathrm{CN}$ stretch mode.

modes $\left(2080-2071 \mathrm{~cm}^{-1}\right)$. Thus, analysis of quantum beats may allow unambiguous assignment of sets of signals to individual redox-structural states of the active site, even in complex (catalytic) mixtures. This is best achieved by Fourier analysis of the coherent decay curves, which yields well-defined frequency components for each pair of coherently excited vibrations (Fig. 5B). This finding is highly relevant, e.g., for the detailed analysis of the $\mathrm{CN}$ stretching vibrations of [NiFe] hydrogenases, since these modes are valuable structural markers but typically hard to analyse due to weak intensity and strong overlap..$^{3,6,21,23}$ Quantum beat analysis can circumvent this difficulty by revealing if (and to which degree) the splitting between the two CN stretch modes differs among constituents of a complex mixture. This may allow assignment of individual species ad hoc or provide physically grounded boundary conditions for otherwise ambiguous global band fit analyses of the IR spectra. The observation of quantum beats also implies a common vibrational ground state, ${ }^{46-48}$ revealing that the two $\mathrm{CN}$ stretch modes of [NiFe] hydrogenases are anharmonically coupled.§

To obtain additional information on the interactions between the $\mathrm{CO}$ and $\mathrm{CN}$ stretching modes, 2D-IR spectra were obtained at a selection of waiting times $T_{\mathrm{w}}$ between pump and probe events. While these spectra were obtained using a Fourier transform (time domain) technique, ${ }^{49-51}$ their general features are best explained using pump-probe (frequency domain) terminology for consistency with earlier results. ${ }^{10,52}$ In contrast to broadband pump-probe spectroscopy, a 2D-IR spectrum provides frequency resolution of both pump and probe events, spreading the information along a second (pump) frequency axis.

Fig. 6 shows the 2D-IR spectrum of oxidized $R e \mathrm{RH}$ in the $\mathrm{Ni}_{\mathrm{a}}$ $\mathrm{S}$ state, split into four quadrants. Diagonal quadrant A exhibits signals resulting from pumping and probing the $\mathrm{CO}$ stretch mode, at $T_{\mathrm{w}}=15 \mathrm{ps}$. The peaks present closely resemble those detected in the pump-probe spectrum at all waiting times (Fig. 3A). Specifically, peak $\mathbf{1}$ corresponds to $\mathbf{0 - 1}$ ground state bleaching and stimulated emission, while signals 2 and 3 can be 


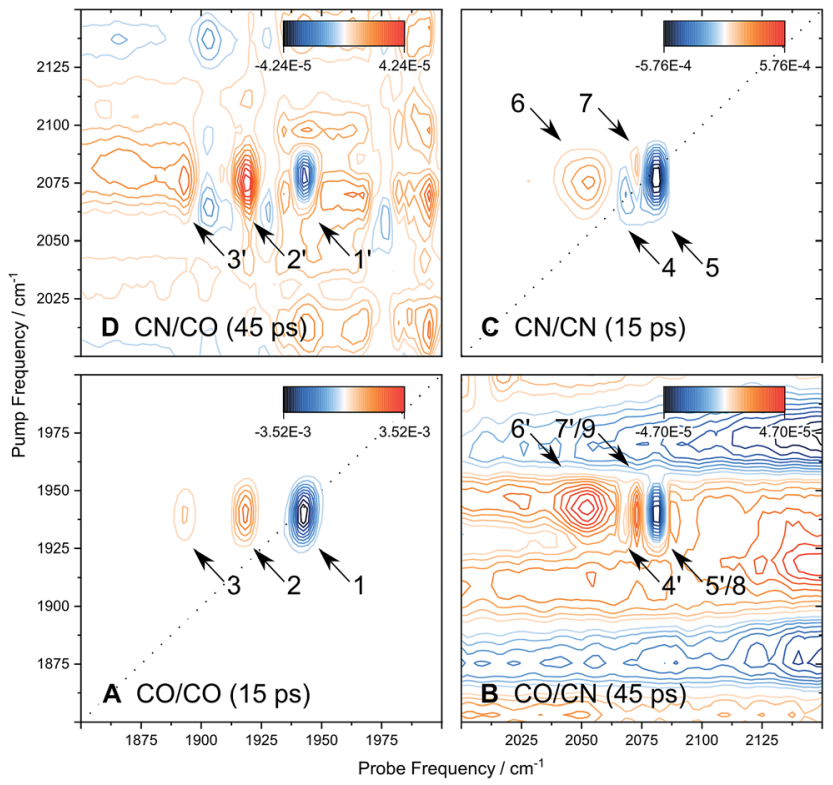

Fig. 6 2D-IR spectrum of oxidized $\mathrm{ReRH}$ in the $\mathrm{Ni}_{\mathrm{a}}-\mathrm{S}$ state, recorded with parallel polarization. Quadrants A to $D$ reflect different pump and probe regimes, as indicated. Waiting times $T_{\mathrm{w}}$ are listed for each quadrant. Signals are numbered, and those reflecting vibrational energy transfer are marked with a prime. For details, see text.

assigned to the 1-2 and 2-3 transient absorption transitions, respectively (Fig. 2). A signal corresponding to the $\mathbf{3}-\mathbf{4}$ transition is also present (Fig. S1 $\dagger$ ) but not visible using the contours shown in Fig. 6A. Due to higher frequency resolution along the probe axis, all signals are slightly ellipsoidal along the pump axis. No significant diagonal elongation of the peaks was observed though, and the diagonal peak shapes were not found to change with $T_{\mathrm{w}}$, indicating that little spectral diffusion is occurring. ${ }^{10,12}$ Inspection of the $\tau$-dependence of the $2 \mathrm{D}$-IR signal arising from the CO stretching mode indicates a 3 ps coherence decay for the 0-1 transition (Fig. 7). Within the homogeneous limit, this decay could be associated with the pure dephasing time $T_{2}^{*}$ since population relaxation is considerably slower $\left(T_{1}=18 \mathrm{ps}\right.$, vide supra $)$. This assignment, however, would imply a homogeneous linewidth of $3.5 \mathrm{~cm}^{-1},{ }^{12,53}$ which is less than the experimental value of $7 \mathrm{~cm}^{-1}$. Likewise, the $10 \mathrm{ps}$ pure dephasing time of the $\mathrm{CN}$ stretch modes (Fig. 5; vide supra) would correspond to a homogeneous width of $1 \mathrm{~cm}^{-1}$, while the experimental value is $6 \mathrm{~cm}^{-1}$. Based on these observations, we conclude that the experimental linewidths reflect moderate inhomogeneous broadening due to a mixture of structurally confined microstates. While fast (multi-scale) dynamics, as observed for a hydrogenase mimic, ${ }^{54}$ cannot be excluded, inhomogeneous distributions of $\leq 7 \mathrm{~cm}^{-1}$ imply that the underlying microstates convert slowly ( $\gg 10 \mathrm{ps}),{ }^{12}$ possibly indicating a multi-minima potential energy surface. Notably, narrow signals in vibrational spectra of hydrogenases have been previously ascribed to conformational constraints imposed by the protein ${ }^{44,55,56}$ which is only valid within the inhomogeneous limit. ${ }^{12,53}$ Based on the high time resolution of the non-linear IR methods employed here, we can verify this implicit assumption,

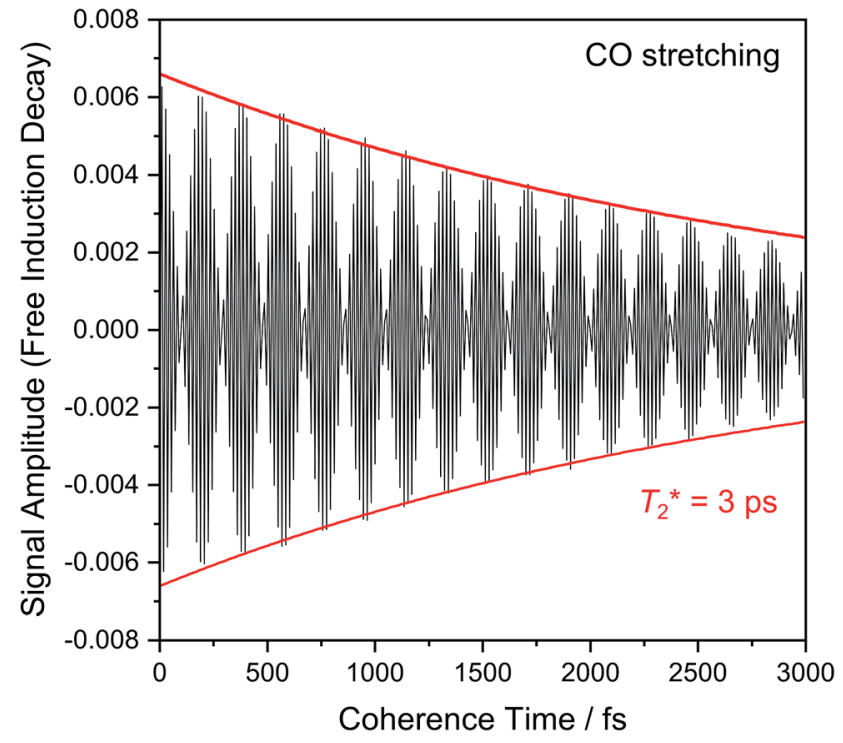

Fig. 7 Evolution of 2D-IR signal intensity (black line) as a function of coherence time $\tau$, modelled as a monoexponential decay curve (red lines). Data refer to the CO stretching mode (probe frequency: $1943 \mathrm{~cm}^{-1}$ ).

demonstrating that hydrogenase active sites are indeed geometrically tuned by the protein matrix. Notably, such a situation has been proposed to be catalytically relevant for efficient $\mathrm{H}_{2}$ binding to the central $\mathrm{Ni}_{\mathrm{a}}$-S intermediate studied here. $^{13,57}$

Signals in diagonal quadrant C of Fig. 6 reflect transitions that arise from the two $\mathrm{CN}$ stretch vibrations $\left(T_{\mathrm{w}}=15 \mathrm{ps}\right)$. Again, these signals resemble those in the pump-probe spectrum (Fig. 3B), but additional information can be extracted. Signals $\mathbf{4}$ and $\mathbf{5}$ can be assigned to the $\mathbf{0 - 1}$ transitions of the asymmetric and symmetric $\mathrm{CN}$ stretch mode, respectively (Fig. 2). The latter feature is elongated towards lower pump frequencies at all waiting times, indicating the presence of a cross peak related to anharmonic coupling between the two $\mathrm{CN}$ modes, ${ }^{10,12}$ in line with a shared vibrational ground state (vide supra) ${ }^{46-48}$ At long waiting times $\left(T_{\mathrm{w}}=20 \mathrm{ps}\right)$, peaks 4 and 5 extend towards higher and lower pump frequencies, respectively, in 2D-IR spectra recorded with perpendicular polarization (Fig. S3 $\dagger$ ). This observation reveals the presence of energy transfer between the two $\mathrm{CN}$ stretch modes, ${ }^{10,12}$ and the observed polarization dependence indicates an orthogonal arrangement of their transition dipole moments, as expected for symmetric and asymmetric modes arising from two CN oscillators. Signal 6 corresponds to the broad continuous feature observed in the pump-probe spectrum (Fig. 3B). Interestingly, this signal covers an extended range of pump frequencies as well, and the apparent band maximum shifts to higher probe frequencies as a function of $T_{\mathrm{w}}$ (Fig. S4 $\dagger$ ), indicating contributions from multiple excited state transitions. According to Fig. S4, $\dagger$ our observations are consistent with contributions from 2-3 and 3-4 transitions of the symmetric stretch mode and the 1-2 transition of the asymmetric stretch mode. In this respect, it should also be noted that the 
vibrational potentials of the $\mathrm{CN}$ stretching modes are likely different from that of the CO stretching vibration. Specifically, the Morse potential is clearly unsuited to describe asymmetric bond stretching, and transition energies may decrease more drastically with increasing quantum numbers than expected from this model. ${ }^{27}$ Furthermore, the anharmonicity of the symmetric stretch mode seems to be underestimated in the pump-probe spectrum, as indicated by strong overlap and partial cancelling of signals 4 and 7 in the 2D-IR spectrum (Fig. 6C). While additional effects cannot be categorically ruled out, ${ }^{37}$ these aspects explain why higher excited state transitions of the $\mathrm{CN}$ stretch modes give rise to an unexpectedly broad and unresolved feature rather than well-defined sharp peaks, as observed for CO stretching (Fig. 3A and 6A). Given the lack of a second frequency axis, all this information is inaccessible from the pump-probe data, highlighting the importance of $2 \mathrm{D}$ IR spectroscopy for disentangling complex vibrational signatures of hydrogenases.

We next turn to the off-diagonal quadrants of the 2D-IR spectrum, B and D, which reflect interactions between $\mathrm{CO}$ and CN stretching modes. Specifically, quadrant B (D) reflects changes in $\mathrm{CN}$ (CO) stretch absorbance due to pumping the $\mathrm{CO}$ (CN) stretch modes. At long waiting times $\left(T_{\mathrm{w}}=45 \mathrm{ps}\right)$, quadrant $\mathrm{D}$ exhibits signals $\mathbf{1}^{\prime}, \mathbf{2}^{\prime}$, and $\mathbf{3}^{\prime}$ that reproduce diagonal features 1, 2, and 3 (Fig. 6A). Being absent at early waiting times (data not shown), these off-diagonal features can be ascribed to vibrational energy transfer from the $\mathrm{CN}^{-}$ligands towards the CO ligand, ${ }^{10,12}$ and the reverse process can be inferred from signals $4^{\prime}, 5^{\prime}, 6^{\prime}$, and $7^{\prime}$ in quadrant $\mathrm{B}$. This confirms that energy from the excited CO stretch mode may be (covalently) dissipated towards the $\mathrm{CN}^{-}$ligands and vice versa. Interestingly, negative signals 8 and 9, coinciding with $5^{\prime}$ and $7^{\prime}$, can be observed from earliest waiting times on, indicating that there is anharmonic coupling between $\mathrm{CO}$ and (symmetric) CN stretch modes (Fig. S2 $\dagger$ ). ${ }^{\mathbf{1 0 , 1 2}}$ Due to the larger transition dipole moment of the pumped CO stretch mode, signal intensities in quadrant B approach those of diagonal CN stretch signals 4, 5, 6, and 7 . According to the projection slice theorem, ${ }^{12}$ these off-diagonal features may therefore contribute to the broadband pumpprobe data in a nonnegligible manner, which explains the kinetic complexity observed for $\mathrm{CN}$ stretch signals in these spectra (vide supra). Considering that signals $4^{\prime}, 5^{\prime}, 6^{\prime}$, and $7^{\prime}$ grow into the 2D-IR spectra as a function of $T_{\mathrm{w}}$, apparent vibrational lifetimes of the $\mathrm{CN}$ stretch modes - as determined from pump-probe spectra - likely represent upper limits. In total, pump-probe and 2D-IR data indicate that $\mathrm{CO}$ and $\mathrm{CN}$ stretch vibrations relax comparably fast within few tens of picoseconds, indicating that energy from both modes is efficiently dissipated via similar (covalent) pathways towards the protein matrix.

\section{Conclusions and outlook}

In the current account, we have demonstrated how ultrafast and multi-dimensional IR techniques can be used to obtain detailed insights into the structure and dynamics of hydrogenases. Utilizing $\mathrm{CO} / \mathrm{CN}$ stretch vibrations as ideal IR probes, these methods were shown to provide access to several unexplored observables that can be used as novel vibrational markers to study the active sites of these enzymes, as summarized in the following.

(1) Using an $\mathrm{O}_{2}$-tolerant [NiFe] hydrogenase as a model system, we have demonstrated that transitions between multiple excited vibrational states can be used to extract detailed information on $\mathrm{CO}$ bond properties that are directly linked to fundamental physical quantities and inaccessible by other experimental techniques (Fig. 4). This approach will assist in understanding how CO bonding controls catalytic $\mathrm{H}_{2}$ binding and cleavage, ${ }^{29,30}$ as exemplified for the $\mathrm{Ni}_{\mathrm{a}}$-S state, the $\mathrm{H}_{2}$ accepting intermediate of [NiFe] hydrogenases. Notably, this analysis also revealed that the harmonic frequency is clearly higher than the experimental value. This supports the previous notion that relative rather than absolute frequencies should be analysed in theoretical studies on, e.g., hydrogenases. ${ }^{23}$ Moreover, the experimental frequency should not be interpreted as a quantitative bond strength measure since the simple relation between this observable and the bond force constant is strictly valid within the harmonic approximation only (see SI $2 \dagger$ ). (2) Analysing the coherence decay of $\mathrm{CO}$ and $\mathrm{CN}$ stretch modes (Fig. 5 and 7), we have gained insights into the magnitude and time scales of equilibrium fluctuations, thereby revealing a structurally confined environment for both types of ligands. Given the high sensitivity of their stretching frequencies towards structural features of the entire [NiFe] centre,,$^{\mathbf{3 , 6 , 2 1 , 2 3}}$ this finding shows that hydrogenase active sites are geometrically tuned by protein structural constraints, as claimed to be mandatory for $\mathrm{H}_{2}$ binding to $\mathrm{Ni}_{\mathrm{a}}-\mathrm{S} .^{13,57}$ Similar analyses promise to yield detailed insights into the structural plasticity of native and artificial maturation intermediates, whose vibrational linewidths are typically much broader than those of mature hydrogenase active sites. ${ }^{\mathbf{4 4 5 5 , 5 6 , 5 8}}$ (3) In principle, CN stretch frequencies are highly sensitive probes, e.g., for structural changes affecting the equatorial plane of the Fe site in [NiFe] hydrogenases. ${ }^{21,23}$ In these enzymes, however, their assignment and interpretation is typically complicated due to overlap of closely spaced low-intensity signals from all probed intermediates in a (catalytic) mixture of states. We have shown that such challenges can be addressed by quantum beat analysis, which allows an unambiguous assignment of $\mathrm{CN}$ stretch modes (Fig. 5). This approach is further facilitated by cross peaks in the 2D-IR spectra, which clearly correlate $\mathrm{CO}$ and $\mathrm{CN}$ stretch signals corresponding to a single redox-structural state (Fig. 6). (4) These cross peaks also provide insights into the extensive interplay between individual vibrations, thereby helping to properly understand the $\mathrm{CO} / \mathrm{CN}$ modes that have been utilized as structural markers for decades (Fig. 2). Specifically, all three stretch modes of [NiFe] hydrogenases are found to be anharmonically coupled, and energy can be quickly transferred both between them and towards the protein matrix, probably via a covalent route involving the bridging cysteine ligands. This finding is expected to be important for energy dissipation during fast, non-classical catalytic steps with notable $\mathrm{CO} / \mathrm{CN}$ bond rearrangement, e.g. $\mathrm{H}_{2}$ binding and cleavage. (5) In the future, 2D-IR cross peaks may also be utilized to gain direct 
structural insight beyond the diatomic ligands by analysing coupling and energy transfer with other marker vibrations that cannot be directly probed in IR absorption experiments. This approach may be further facilitated by the intrinsic sensitivity of many introduced observables to solvent, substrate, or protein isotope exchanges, which is expected to inform on, e.g., hydrogen and oxygen adducts. This way, the structural analysis of $\mathrm{CO} / \mathrm{CN}$ stretch modes can be rationalized and expanded without losing the intrinsic advantages of these highly localized reporter vibrations.

Despite the plethora of additional information available from nonlinear techniques, they retain the intrinsic advantages of IR spectroscopy, i.e. various sample forms can be probed in a non-invasive manner under physiologically relevant conditions and versatile experimental control. Moreover, sample requirements and acquisition times (see Experimental details) are able to compete with benchtop IR experiments, in contrast to other advanced (vibrational) spectroscopies. Remarkably, many of the outlined features were also observed for lowconcentration samples that appeared almost IR-transparent in basic absorption experiments. This is due to the fact that the nonlinear nature of the 2D-IR signal amplifies narrow transitions with large extinction coefficients. ${ }^{59}$ Thus, nonlinear IR techniques are particularly suited for in vivo studies ${ }^{\mathbf{2 0 , 2 2 , 2 3 , 6 0}}$ that are highly informative but often limited by the low hydrogenase content in living organisms. Such studies would particularly benefit from the sensitivity of 2D-IR spectroscopy towards solvent effects, intermolecular interactions, and other environmental factors. ${ }^{\mathbf{1 0}}$

While demonstrated for [NiFe] hydrogenases, the outlined approaches can be easily adapted for the investigation of [FeFe] and [Fe] hydrogenases, $\mathrm{CO} / \mathrm{CN}$-bound maturation factors, and other enzymes or (bioinspired) catalysts that contain or interact with feasible IR-chromophores, e.g. nitrogenase, CO dehydrogenase, and NO reductase. Finally, we stress the exceptional time resolution of non-linear IR techniques, which exceeds all previous studies on hydrogenases by at least three orders of magnitude. Notably, this valuable trait is not limited to equilibrium studies but can also be utilized in nonequilibrium experiments. To this end, both techniques can be combined with an additional (visible) pulse, which allows triggering and probing entire reactions or individual elementary steps with ultra-high time resolution. This trait is of utmost importance for probing fast and so-far inaccessible catalytic events including $\mathrm{H}_{2}$ binding and cleavage, and even non-stationary structures along catalytic reaction coordinates come into reach.

\section{Experimental details}

\section{Preparation of $\mathrm{ReRH}$}

R. eutropha HF574 (pGE\#3888), ${ }^{\mathbf{1 3 2 4 , 6 1}}$ overproducing doubletagged native (double-dimeric) $R e \mathrm{RH}$, was cultivated heterotrophically in a mineral salt medium, as described previously. ${ }^{\mathbf{1 3 , 6 2}}$ After cell harvest and disruption, $R e \mathrm{RH}$ was purified from the soluble extract according to established procedures. ${ }^{13,17,24}$ For spectroscopic studies, double-dimeric $R e \mathrm{RH}$ was ultrafiltrated (Amicon Ultra-100, Millipore) to a final concentration of $c a$.
$0.6 \mathrm{mM}$ (corresponding to a [NiFe] centre concentration of 1.2 $\mathrm{mM}$ ) and stored at $-80{ }^{\circ} \mathrm{C}$ until further usage.

\section{Ultrafast IR pump-probe and 2D-IR spectroscopy}

Spectra were recorded in transmission mode using a gas-tight and temperature-controlled $\left(T=10{ }^{\circ} \mathrm{C}\right)$ small-volume sandwich cell (optical path length $=50 \mu \mathrm{m}, V \approx 8 \mu \mathrm{L}$ ) equipped with $\mathrm{CaF}_{2}$ windows. All data were acquired in (pseudo) pump probe geometry utilizing mid-IR pulses (centre frequency = $2000 \mathrm{~cm}^{-1}$; bandwidth $>300 \mathrm{~cm}^{-1}$; pulse duration $=50 \mathrm{fs}$; repetition rate $=10 \mathrm{kHz}$ ) from the ULTRA laser-system as described previously. ${ }^{49-51,63}$ Series of pump-probe spectra (accumulation time $=3 \mathrm{~s}$ ) were recorded by scanning the pump-probe delay time from -10 to $54 \mathrm{ps}$ (step size $=250 \mathrm{fs}$ ).

2D-IR spectra (accumulation time $=300 \mathrm{~s}$ ) were obtained at several selected waiting times $T_{\mathrm{w}}$ between 250 fs and 50 ps. For each fixed $T_{\mathrm{w}}, 2 \mathrm{D}$-IR data were obtained in a time-domain fashion by scanning the coherence time $\tau$ between two pulseshaper generated collinear pump pulses from 0 to 2997 fs (step size $9 \mathrm{fs}$ ) prior to overlap with the probe pulse and selfheterodyned detection of the collinearly emitted signal. ${ }^{49-51}$ The pump frequency axis was obtained by Fourier transformation of the time-domain signal with respect to $\tau$, while the probe frequency axis of both 2D-IR and pump-probe spectra was obtained by signal dispersion in a spectrograph and detection via liquid-nitrogen cooled 128-element MCT (mercury-cadmium-telluride) detectors with a resolution of $<2 \mathrm{~cm}^{-1}$. Four-frame phase cycling was employed in 2D-IR data acquisition to limit contributions from pump light scattered on the detector. ${ }^{50}$

\section{Conflicts of interest}

There are no conflicts to declare.

\section{Acknowledgements}

The authors thank the Leverhulme Trust (RPG-2018-188) and the DFG (Cluster of Excellence UniSysCat) for financial support and the STFC for funding access to ULTRA.

\section{Notes and references}

$\ddagger$ Pump-probe polarization geometries (parallel/perpendicular/magic-angle) refer to the angle between electric field vectors of the two pulses. Due to the huge size of native, double-dimeric $R e \mathrm{RH}(177.8 \mathrm{kDa}),{ }^{17}$ molecular rotation on the timescale of the IR pump-probe experiments is assumed to be negligible. Thus, analysis of vibrational lifetimes does not require spectra recorded under magic angle polarization per se (see SI3†).

$\S$ Notably, anharmonic coupling between symmetric and asymmetric stretch modes should not be confused with the well-known coupled stretching of the two $\mathrm{CN}$ bonds within both modes. ${ }^{5}$ While the latter aspect is well captured by harmonic vibrational analyses (see, e.g., ref. 13, 19 and 21), the experimental observation of anharmonic coupling illustrates a partial breakdown of the harmonic approximation.

1 Hydrogen as a Fuel: Learning from Nature, R. Cammack, M. Frey and R. Robson, Taylor and Francis, London, 2001. 
2 (a) K. A. Bagley, C. J. van Garderen, M. Chen, E. C. Duin, S. P. J. Albracht and W. H. Woodruff, Biochemistry, 1994, 33, 9229; (b) A. Volbeda, E. Garcin, C. Piras, A. L. de Lacey, V. M. Fernandez, E. C. Hatchikian, M. Frey and J. C. Fontecilla-Camps, J. Am. Chem. Soc., 1996, 118, 12989; (c) A. J. Pierik, M. Hulstein, W. R. Hagen and S. P. J. Albracht, Eur. J. Biochem., 1998, 258, 572; (d) T. M. van der Spek, A. F. Arendsen, R. P. Happe, S. Yun, K. A. Bagley, D. J. Stufkens, W. R. Hagen and S. P. J. Albracht, Eur. J. Biochem., 1996, 237, 629.

3 K. A. Bagley, E. C. Duin, W. Roseboom, S. P. J. Albracht and W. H. Woodruff, Biochemistry, 1995, 34, 5527.

4 R. P. Happe, W. Roseboom, A. J. Pierik, S. P. J. Albracht and K. A. Bagley, Nature, 1997, 385, 126.

5 A. J. Pierik, W. Roseboom, R. P. Happe, K. A. Bagley and S. P. J. Albracht, J. Biol. Chem., 1999, 274, 3331.

6 M. Y. Darensbourg, E. J. Lyon and J. J. Smee, Coord. Chem. Rev., 2000, 206-207, 533.

7 W. Lubitz, H. Ogata, O. Rüdiger and E. Reijerse, Chem. Rev., 2014, 114, 4081.

8 A. L. de Lacey, V. M. Fernandez, M. Rousset and R. Cammack, Chem. Rev., 2007, 107, 4304.

9 (a) B. L. Greene, C.-H. Wu, P. M. McTernan, M. W. W. Adams and R. B. Dyer, J. Am. Chem. Soc., 2015, 137, 4558; (b) B. L. Greene, G. E. Vansuch, C.-H. Wu, M. W. W. Adams and R. B. Dyer, J. Am. Chem. Soc., 2016, 138, 13013; (c) B. L. Greene, C.-H. Wu, G. E. Vansuch, M. W. W. Adams and R. B. Dyer, Biochemistry, 2016, 55, 1813; (d) S. J. George, S. Kurkin, R. N. Thorneley and S. P. J. Albracht, Biochemistry, 2004, 43, 6808; (e) S. Kurkin, S. J. George, R. N. Thorneley and S. P. J. Albracht, Biochemistry, 2004, 43, 6820; ( $f$ ) B. L. Greene, G. J. Schut, M. W. W. Adams and R. B. Dyer, ACS Catal., 2017, 7, 2145.

10 N. T. Hunt, Chem. Soc. Rev., 2009, 38, 1837.

11 N. T. Hunt, J. A. Wright and C. Pickett, Inorg. Chem., 2016, 55, 399.

12 P. Hamm and M. T. Zanni, Concepts and Methods of $2 D$ Infrared Spectroscopy, Cambridge University Press, Cambridge, United Kingdom, 2011.

13 M. Horch, J. Schoknecht, M. A. Mroginski, O. Lenz, P. Hildebrandt and I. Zebger, J. Am. Chem. Soc., 2014, 136, 9870.

14 F. Roncaroli, E. Bill, B. Friedrich, O. Lenz, W. Lubitz and M.-E. Pandelia, Chem. Sci., 2015, 6, 4495.

15 (a) A. J. Healy, P. A. Ash, O. Lenz and K. A. Vincent, Phys. Chem. Chem. Phys., 2013, 15, 7055; (b) M. Brecht, M. van Gastel, T. Buhrke, B. Friedrich and W. Lubitz, J. Am. Chem. Soc., 2003, 125, 13075.

16 A. J. Pierik, M. Schmelz, O. Lenz, B. Friedrich and S. P. J. Albracht, FEBS Lett., 1998, 438, 231.

17 M. Bernhard, T. Buhrke, B. Bleijlevens, A. L. de Lacey, V. M. Fernandez, S. P. J. Albracht and B. Friedrich, J. Biol. Chem., 2001, 276, 15592.

18 T. Buhrke, S. Löscher, O. Lenz, E. Schlodder, I. Zebger, L. K. Andersen, P. Hildebrandt, W. Meyer-Klaucke, H. Dau, B. Friedrich and M. Haumann, J. Biol. Chem., 2005, 280, 19488.
19 M. Horch, Y. Rippers, M. A. Mroginski, P. Hildebrandt and I. Zebger, ChemPhysChem, 2013, 14, 185.

20 M. Horch, L. Lauterbach, M. Saggu, P. Hildebrandt, F. Lendzian, R. Bittl, O. Lenz and I. Zebger, Angew. Chem., Int. Ed., 2010, 49, 8026.

21 M. Horch, L. Lauterbach, M. A. Mroginski, P. Hildebrandt, O. Lenz and I. Zebger, J. Am. Chem. Soc., 2015, 137, 2555.

22 M. Horch, L. Lauterbach, O. Lenz, P. Hildebrandt and I. Zebger, FEBS Lett., 2012, 586, 545.

23 M. Horch, P. Hildebrandt and I. Zebger, Phys. Chem. Chem. Phys., 2015, 17, 18222.

24 T. Buhrke, O. Lenz, N. Krauss and B. Friedrich, J. Biol. Chem., 2005, 280, 23791.

25 B. Bleijlevens, F. A. van Broekhuizen, A. L. de Lacey, W. Roseboom, V. M. Fernandez and S. P. J. Albracht, J. Biol. Inorg Chem., 2004, 9, 743.

26 S. M. Arrivo, T. P. Dougherty, W. T. Grubbs and E. J. Heilweil, Chem. Phys. Lett., 1995, 235, 247.

27 T. Witte, J. S. Yeston, M. Motzkus, E. J. Heilweil and K.-L. Kompa, Chem. Phys. Lett., 2004, 392, 156.

28 P. M. Morse, Phys. Rev., 1929, 34, 57.

29 G. J. Kubas, Chem. Rev., 2007, 107, 4152.

30 F. A. Armstrong and J. C. Fontecilla-Camps, Science, 2008, 321, 498.

31 (a) M. Winkler, M. Senger, J. Duan, J. Esselborn, F. Wittkamp, E. Hofmann, U.-P. Apfel, S. T. Stripp and T. Happe, Nat. Commun., 2017, 8, 16115; (b) D. W. Mulder, M. W. Ratzloff, M. Bruschi, C. Greco, E. Koonce, J. W. Peters and P. W. King, J. Am. Chem. Soc., 2014, 136, 15394; (c) E. J. Reijerse, C. C. Pham, V. Pelmenschikov, R. Gilbert-Wilson, A. Adamska-Venkatesh, J. F. Siebel, L. B. Gee, Y. Yoda, K. Tamasaku, W. Lubitz, T. B. Rauchfuss and S. P. Cramer, J. Am. Chem. Soc., 2017, 139, 4306.

32 (a) K. Adamczyk, N. Simpson, G. M. Greetham, A. Gumiero, M. A. Walsh, M. Towrie, A. W. Parker and N. T. Hunt, Chem. Sci., 2015, 6, 505; (b) P. Hamm, M. Lim and R. M. Hochstrasser, J. Chem. Phys., 1997, 107, 10523.

33 K. Ohta, H. Maekawa and K. Tominaga, Chem. Phys. Lett., 2004, 386, 32.

34 P. Yu, F. Yang, J. Zhao and J. Wang, J. Phys. Chem. B, 2014, 118, 3104.

35 J. T. King, M. R. Ross and K. J. Kubarych, J. Phys. Chem. B, 2012, 116, 3754.

36 J. T. Fourkas, H. Kawashima and K. A. Nelson, J. Chem. Phys., 1995, 103, 4393.

37 P. W. J. M. Frederix, K. Adamczyk, J. A. Wright, T. Tuttle, R. V. Ulijn, C. J. Pickett and N. T. Hunt, Organometallics, 2014, 33, 5888.

38 R. Kania, P. W. J. M. Frederix, J. A. Wright, R. V. Ulijn, C. J. Pickett and N. T. Hunt, J. Chem. Phys., 2012, 136, 44521.

39 G. M. Bonner, A. R. Ridley, S. K. Ibrahim, C. J. Pickett and N. T. Hunt, Faraday Discuss., 2010, 145, 429.

40 S. Kaziannis, J. A. Wright, M. Candelaresi, R. Kania, G. M. Greetham, A. W. Parker, C. J. Pickett and N. T. Hunt, Phys. Chem. Chem. Phys., 2011, 13, 10295. 
41 A. I. Stewart, I. P. Clark, M. Towrie, S. K. Ibrahim, A. W. Parker, C. J. Pickett and N. T. Hunt, J. Phys. Chem. B, 2008, 112, 10023.

42 V. M. Kenkre, A. Tokmakoff and M. D. Fayer, J. Chem. Phys., 1994, 101, 10618.

43 (a) J. R. Hill, A. Tokmakoff, K. A. Peterson, B. Sauter, D. Zimdars, D. D. Dlott and M. D. Fayer, J. Phys. Chem., 1994, 98, 11213; (b) J. R. Hill, D. D. Dlott, M. D. Fayer, K. A. Peterson, C. W. Rella, M. M. Rosenblatt, K. S. Suslick and C. J. Ziegler, Chem. Phys. Lett., 1995, 244, 218; (c) J. R. Hill, D. D. Dlott, C. W. Rella, K. A. Peterson, S. M. Decatur, S. G. Boxer and M. D. Fayer, J. Phys. Chem., 1996, 100, 12100; (d) J. R. Hill, D. D. Dlott, C. W. Rella, T. I. Smith, H. A. Schwettman, K. A. Peterson, A. Kwok, K. D. Rector and M. D. Fayer, Biospectroscopy, 1996, 2, 277; (e) J. R. Hill, C. J. Ziegler, K. S. Suslick, D. D. Dlott, C. W. Rella and M. D. Fayer, J. Phys. Chem., 1996, 100, 18023; (f) D. D. Dlott, M. D. Fayer, J. R. Hill, C. W. Rella, K. S. Suslick and C. J. Ziegler, J. Am. Chem. Soc., 1996, 118, 7853; $(g)$ J. C. Owrutsky, M. Li, B. Locke and R. M. Hochstrasser, J. Phys. Chem., 1995, 99, 4842.

44 S. Katz, J. Noth, M. Horch, H. S. Shafaat, T. Happe, P. Hildebrandt and I. Zebger, Chem. Sci., 2016, 7, 6746.

45 (a) S. Kamali, H. Wang, D. Mitra, H. Ogata, W. Lubitz, B. C. Manor, T. B. Rauchfuss, D. Byrne, V. Bonnefoy, F. E. Jenney Jr, M. W. Adams, Y. Yoda, E. E. Alp, J. Zhao and S. P. Cramer, Angew. Chem., Int. Ed., 2013, 52, 724; (b) L. Lauterbach, H. Wang, M. Horch, L. B. Gee, Y. Yoda, Y. Tanaka, I. Zebger, O. Lenz and S. P. Cramer, Chem. Sci., 2015, 6, 1055; (c) E. Siebert, M. Horch, Y. Rippers, J. Fritsch, S. Frielingsdorf, O. Lenz, E. F. Velazquez, F. Siebert, L. Paasche, U. Kuhlmann, F. Lendzian, M. A. Mroginski, I. Zebger and P. Hildebrandt, Angew. Chem., Int. Ed., 2013, 52, 5162; (d) J. M. Kuchenreuther, Y. Guo, H. Wang, W. K. Myers, S. J. George, C. A. Boyke, Y. Yoda, E. E. Alp, J. Zhao, R. D. Britt, J. R. Swartz and S. P. Cramer, Biochemistry, 2013, 52, 818; (e) Y. Guo, H. Wang, Y. Xiao, S. Vogt, R. K. Thauer, S. Shima, P. I. Volkers, T. B. Rauchfuss, V. Pelmenschikov, D. A. Case, E. E. Alp, W. Sturhahn, Y. Yoda and S. P. Cramer, Inorg. Chem., 2008, 47, 3969.

46 H. Bitto and J. R. Huber, Opt. Commun., 1990, 80, 184.

47 R. T. Carter and J. R. Huber, Chem. Soc. Rev., 2000, 29, 305. 48 E. Hack and J. R. Huber, Int. Rev. Phys. Chem., 1991, 10, 287. 49 L. P. DeFlores, R. A. Nicodemus and A. Tokmakoff, Opt. Lett., 2007, 32, 2966.

50 S.-H. Shim, D. B. Strasfeld, Y. L. Ling and M. T. Zanni, Proc. Natl. Acad. Sci. U. S. A., 2007, 104, 14197.

51 S.-H. Shim and M. T. Zanni, Phys. Chem. Chem. Phys., 2009, 11, 748.
52 (a) P. Hamm, M. Lim and R. M. Hochstrasser, J. Phys. Chem. $B$, 1998, 102, 6123; (b) V. Cervetto, J. Helbing, J. Bredenbeck and P. Hamm, J. Chem. Phys., 2004, 121, 5935.

53 R. Kubo, A Stochastic Theory of Line Shape, in Advances in Chemical Physics: Stochastic Processes in Chemical Physics, ed. K. E. Shuler, John Wiley \& Sons Inc., Hoboken, New Jersey, 1969, vol. 15, pp. 101-127.

54 P. A. Eckert and K. J. Kubarych, J. Phys. Chem. A, 2017, 121, 608.

55 J. Esselborn, C. Lambertz, A. Adamska-Venkatesh, T. Simmons, G. Berggren, J. Noth, J. Siebel, A. Hemschemeier, V. Artero, E. Reijerse, M. Fontecave, W. Lubitz and T. Happe, Nat. Chem. Biol., 2013, 9, 607.

56 G. Berggren, A. Adamska, C. Lambertz, T. R. Simmons, J. Esselborn, M. Atta, S. Gambarelli, J. M. Mouesca, E. Reijerse, W. Lubitz, T. Happe, V. Artero and M. Fontecave, Nature, 2013, 499, 66.

57 M. Bruschi, M. Tiberti, A. Guerra and L. de Gioia, J. Am. Chem. Soc., 2014, 136, 1803.

58 (a) I. Burstel, E. Siebert, G. Winter, P. Hummel, I. Zebger, B. Friedrich and O. Lenz, J. Biol. Chem., 2012, 287, 38845; (b) B. Soboh, S. T. Stripp, E. Muhr, C. Granich, M. Braussemann, M. Herzberg, J. Heberle and S. R. Gary, FEBS Lett., 2012, 586, 3882; (c) B. Soboh, S. T. Stripp, C. Bielak, U. Lindenstrauss, M. Braussemann, M. Javaid, M. Hallensleben, C. Granich, M. Herzberg, J. Heberle and R. G. Sawers, FEBS Lett., 2013, 587, 2512; (d) S. T. Stripp, B. Soboh, U. Lindenstrauss, M. Braussemann, M. Herzberg, D. H. Nies, R. G. Sawers and J. Heberle, Biochemistry, 2013, 52, 3289; (e) S. T. Stripp, U. Lindenstrauss, R. G. Sawers and B. Soboh, PLoS One, 2015, 10, e0133118; $(f)$ S. T. Stripp, U. Lindenstrauss, C. Granich, R. G. Sawers and B. Soboh, PLoS One, 2014, 9, e107488.

59 M. Grechko and M. T. Zanni, J. Chem. Phys., 2012, 137, 184202.

60 L. Lauterbach, J. Liu, M. Horch, P. Hummel, A. Schwarze, M. Haumann, K. A. Vincent, O. Lenz and I. Zebger, Eur. J. Inorg. Chem., 2011, 2011, 1067.

61 L. Kleihues, O. Lenz, M. Bernhard, T. Buhrke and B. Friedrich, J. Bacteriol., 2000, 182, 2716.

62 (a) T. Goris, A. F. Wait, M. Saggu, J. Fritsch, N. Heidary, M. Stein, I. Zebger, F. Lendzian, F. A. Armstrong, B. Friedrich and O. Lenz, Nat. Chem. Biol., 2011, 7, 310; (b) M. Ludwig, J. A. Cracknell, K. A. Vincent, F. A. Armstrong and O. Lenz, J. Biol. Chem., 2009, 284, 465.

63 G. M. Greetham, P. Burgos, Q. Cao, I. P. Clark, P. S. Codd, R. C. Farrow, M. W. George, M. Kogimtzis, P. Matousek, A. W. Parker, M. R. Pollard, D. A. Robinson, Z.-J. Xin and M. Towrie, Appl. Spectrosc., 2010, 64, 1311. 